EOMmUn Communication et organisation

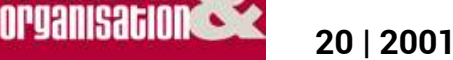

La communication du risque

\title{
La communication du risque
}

Valérie Carayol et Gino Gramaccia

\section{OpenEdition}

Journals

Édition électronique

URL : http://journals.openedition.org/communicationorganisation/2547

DOI : 10.4000/communicationorganisation.2547

ISSN : 1775-3546

Éditeur

Presses universitaires de Bordeaux

Édition imprimée

Date de publication : 1 novembre 2001

ISSN : 1168-5549

\section{Référence électronique}

Valérie Carayol et Gino Gramaccia, «La communication du risque », Communication et organisation [En ligne], 20 | 2001, mis en ligne le 27 mars 2012, consulté le 01 mai 2019. URL : http://

journals.openedition.org/communicationorganisation/2547; DOI : 10.4000/

communicationorganisation.2547

Ce document a été généré automatiquement le 1 mai 2019.

(c) Presses universitaires de Bordeaux 


\title{
La communication du risque
}

\author{
Valérie Carayol et Gino Gramaccia
}

1 La question du risque doit être posée globalement : aucune discipline ne peut, à elle seule, être propriétaire d'un objet réfractaire par nature aux lois positives qui gouvernent, en principe, les phénomènes naturels ou les processus achevés. Au plan le plus général, ce qui anime les chercheurs et les praticiens, c'est, à propos du risque, la problématique du désordre, sa probabilité et sa logique et, au final, la possibilité d'en prévenir les causes ou, au mieux, d'en réduire les effets. C'est sans doute parce quelque chose résiste toujours aux effets de sa gestion que les acteurs qui ont des responsabilités dans ce domaine deviennent particulièrement prolixes. Si la communication prend le relais de la gestion, c'est dans la mesure où on lui accorde des vertus explicatives, préventives, ou au mieux palliatives et conjuratoires. On sait que la maîtrise du risque peut faire l'objet de discours enchanteurs pour la mystification des multiples formes d'affrontement ou d'évitement du risque mortel (dans le management de l'exploit, de la prouesse, de la performance).

2 Mais communiquer à propos du risque engendre d'autres risques ou, pour les médiateurs les plus avertis, d'autres ressources stratégiques qu'il faut savoir exploiter. Pour plusieurs raisons : tant pour ne pas ajouter le risque au risque en abusant du pouvoir dramatisant de l'imaginaire social du danger (dans le domaine du nucléaire civil, par exemple), tant pour produire exactement le contraire : déclencher des peurs quand elles ne se justifient pas, compte tenu de ce que les experts sont supposés savoir du risque de danger (dans l'univers de la Bourse, notamment).

3 La question du risque s'étend aujourd'hui, dans les organisations contemporaines, à toutes les stratégies qui conditionnent leur survie (commerciales, technologiques, financières, sociales). Le risque peut aussi être considéré comme une composante du projet de modernisation des organisations contemporaines dont les modes de structuration (reconfiguration, transversalisation, développement en réseau, fusionacquisition) intègrent la possibilité de l'aléa, la logique de l'événement, la notion de trajectoire critique, la multiplication d'actions locales. Tous ces processus modifient en profondeur la carte des solidarités traditionnelles au profit d'une instrumentation de communication et de gestion qui se met alors au service d'une logique de contrôle: le 
risque devient une menace objective pour la prévention de laquelle l'acteur collectif doit impérativement se résoudre. «En objectivant les menaces, l'instrument de gestion peut en effet simplifier et faire partager à tous des informations qui expliquent la violence des actions managériales", dit David Courpasson (2000). La transparence communicationnelle prônée dans les années 80 bute-t-elle, in fine, sur la question du risque, qui refait advenir dans les questionnements contemporains la question du secret, sous un autre jour que celui du pouvoir?

Le vingtième dossier de Communication \& Organisation traite la problématique communicationnelle du risque et fait écho aux débats engagés dans le dossier $n^{\circ} 16$ sur les relations entre crise et communication ${ }^{1}$.

Dans sa contribution, Ariette Bouzon retrace d'abord l'histoire d'un concept «nomade ». Son sens a puisé à bien des métiers et des disciplines (en droit, en médecine, dans les activités du commerce...) pour se décliner ensuite en trois paradigmes: celui de la responsabilité au $\mathrm{XIX}^{\mathrm{e}}$ siècle, de la solidarité au $\mathrm{XX}^{\mathrm{e}}$ siècle et plus près de nous, celui de précaution. Ce renversement de perspective est plein de sens: il part de l'idée d'un individu responsable de ses actes, prend ensuite les accents de la solidarité, de la prévention et de la protection d'état, s'étend enfin à l'échelle environnementale et planétaire puisqu'il s'agit maintenant de précaution quand la certitude scientifique a laissé place à la notion « d'hypothèse de risque scientifiquement crédible ». Une éthique consumériste, résignée dans l'esprit puisqu'elle accepte une part incompressible de risque, est attentive à la lettre ou à l'étiquette qui devient gage de transparence. Informer en place publique: telle est la voie la plus sûre et la plus respectable pour jouer de prudence. Mais la maîtrise des risques passe par l'exploration concertée de toutes les branches événementielles du cheminement décisionnel. Le risque est affaire d'évaluation, d'estimation, d'exploration en termes de gravité, de criticité, de probabilité. Comment concilier alors le projet d'informer, qui se nourrit de certitudes argumentées au présent et ces effets de perspectives représentées à l'horizon du temps? Il y a bien un moyen de jouer avec le temps : c'est de le remonter en recomposant la genèse d'un produit. La traçabilité refait l'histoire autant juridique que productive des organisations. Elle explicite le respect de la volonté de bien produire dans le cadre des engagements successifs sur la chaîne clients-fournisseurs. Elle est l'élément clé de la représentation du risque et, du côté de l'usager, du client, du citoyen, des victimes potentielles devrionsnous dire, de sa perception : «le véritable enjeu du principe de précaution est peut-être davantage de bien gérer la perception des risques que les risques eux-mêmes ", dit Ariette Bouzon.

6 Cette idée sera reprise par Hervé Courtot. Si la gestion du risque est devenue la fonction majeure de la stratégie de l'entreprise, cette gestion renvoie, comme le souligne l'auteur, à des enjeux de communication. Et de tels enjeux passent par la mobilisation d'équipes vigilantes, par l'incontournable nécessité de coopérer face à ce qui motive et légitime, dans tout projet, la solidarité dans l'action: un environnement complexe, incertain, contingent. Mais la communication dans un contexte de risque n'est pas un simple exercice de transparence. Elle ne se réduit pas à informer, à rendre compte des conditions des risques, à présenter le rapport des aléas-types. Elle doit agir au contraire sur la perception du risque et pour ce faire, l'interpréter de manière à ce que les acteurs du projet l'acceptent comme un défi collectif. C'est précisément sur ce point qu'insiste Hervé Courtot. Le management du risque s'applique à des collectifs. Les travers individuels (recherche du « coupable», méfiance, passivité, résignation, rétention d'information...) 
doivent se "dissoudre» dans l'esprit d'équipe, dans une communauté d'engagement, dans la volonté d'en découdre avec des contraintes objectives. Si l'individu n'a pas sa place dans une telle aventure (disons le profil individuel et les «risques» de déviance dont il est le porteur subjectif), c'est dans la mesure où personne n'est absolument détenteur de certitudes suffisamment fondées pour faire valoir de quelconques prérogatives ou de faux prétextes. Par exemple, dit l'auteur, la capitalisation des connaissances dans un projet " n'a d'intérêt que si elle est collective et pas uniquement individuelle». Finalement la prévention des risques passe par la composition d'un ensemble (toujours un peu idéal, il est vrai) homogène regroupant une équipe solidaire (ce qui est un pléonasme), un dispositif informationnel efficace et une hiérarchie vigilante et engagée.

7 Un tel dispositif, Didier Gourc, Béatrice Vacher et Hervé Pingaud en présentent une approche appliquée à la gestion de projet. Conçu pour susciter la vigilance mutuelle, il s'inscrit dans le cadre plus général d'un management des risques attentif à tous les facteurs et à toutes les composantes du risque. S'il est dit que la vigilance est une question d'organisation, c'est au prix d'une méthodologie tout entière dédiée à la connaissance prospective du déroulement du projet. On entre ainsi dans une logique d'anticipation de processus dont il convient d'évaluer la gravité, la criticité ou, dans le cas positif, la valeur opportune. La vigilance mutuelle, l'intelligence collective et partant, la performance organisationnelle résultent de la construction d'un réseau, laquelle traduit les enjeux du projet selon les types d'acteurs. Un tel réseau doit favoriser la formation d'une solidarité pratique. Mais comment convaincre les individus d'adhérer à une telle cause? On ne parlera pas de manipulation, mais plutôt de contrainte ritualisée, ou encore de communication coercitive qui doit déboucher sur un résultat inversé (et un peu paradoxal: Beauvois et Joule sont cités): faire en sorte que chacun des acteurs s'approprie les raisons du succès. Cette dynamique doit se dérouler dans un climat consensuel au moyen d'une démarche qui permet de dépassionner les débats, de favoriser le comportement de diagnostic, de conduire à l'interprétation objective des faits (ce sont les buts opératoires de la méthode Delphi). Mission difficile si l'on sait que la communication sur le risque peut avoir des effets aggravants, anxiogènes, pathologiques, mais aussi culpabilisants. Les chemins stratégiques sont étroits. S'impose alors l'idée, pour le pilotage de projet, d'une fonction dédiée à la maîtrise des risques, la fonction "Risk Management » dont la mission est de "coordonner la mise en cuvre opérationnelle de la démarche de maîtrise des risques auprès et avec les équipiers ».

8 Une telle mission permet d'élever le risque à la dimension d'une problématique identitaire. Nous sommes dans le cas de configurations productives qui imposent d'identifier, d'objectiver et d'exclure les processus du risque. Le risque est un mauvais objet (c'est le cas le plus général) et dès lors on mesure le bénéfice managérial qu'on peut en retirer si sa prévention devient explicitement un enjeu collectif. Paradoxalement, ce mauvais objet peut révéler une valeur stratégique intrinsèque. Telle est, en substance, la thèse de Dominique Poizat à propos du " produit » «Sports d'hiver ». L'auteur met l'accent sur une stratégie commerciale qui supporte les paradoxes, les cultive même, puisque l'action sportive conjugue, pour la crédibilité de sa politique marketing, toutes sortes de contradictions constitutives : sociologique, économique, juridique, politique, médiatique. Cette problématique de l'hédonisme complexe concentre toutes les difficultés liées aux industries de l'imaginaire, fut-il sportif. La communication, dans ce domaine, doit louvoyer (ce n'est pas le moindre paradoxe) entre au moins deux acceptions de la notion 
de prévention: un sens économique (ne pas effrayer la clientèle) et un sens juridique (s'engager sur la sécurité), voire procédurier. Dans ce cas, l'objectif est faire de la prévention pour la prévention, autant dire pour se couvrir... La conclusion de l'auteur est un réquisitoire contre ce qu'il appelle "l'objectivation des référents du risque", autrement dit la mise en place d'appareils juridiques au service, en dernière instance, de considérations de profit: "le pratiquant devient un «client» dont les modalités de pratiques sont prises en charge " collectivement » ».

Le concept de prévention est aussi au cœur du travail de Denis Réquillart. La réflexion sur ce thème est abordée à l'occasion de l'analyse d'une campagne de communication ministérielle lancée en avril 2000 dans le cadre de la lutte contre les drogues et la toxicomanie, intitulée "Savoir plus, risquer moins ». Innovante à plus d'un titre, cette action de communication publique introduirait des ruptures dans les pratiques traditionnelles de communication sur les risques sanitaires. Elle permet à l'auteur, qui analyse aussi d'un point de vue comparatif, d'autres types de campagnes, notamment dans le champ de la prévention routière, d'introduire d'intéressantes distinctions sur les forces à l'œuvre dans le champ de la communication publique, notamment celles de la communication de prévention et de la communication de santé publique. La première, traditionnellement envisagée dans une perspective éducative serait essentiellement centrée sur l'individu considéré isolément comme responsable de sa santé, en occultant la dimension sociale et politique des phénomènes sanitaires. La communication de santé publique, en diffusant des normes de comportement susceptibles de réduire les risques sanitaires, proposerait un discours public légitime sur lequel la collectivité devrait pouvoir s'appuyer. Elle serait plus axée sur la responsabilité collective et la solidarité inscrites dans l'espace public. De très intéressantes questions sont posées qui nous amènent après cette lecture à nous interroger sur l'existence et l'intérêt d'une distinction entre risque individuel, risque collectif et risque public. Cette analyse d'inscrit dans une réflexion plus globale sur le processus d'industrialisation de la santé.

De prévention, il sera encore question dans l'article de Georges Aubert, qui a mené une étude pour le compte de la Préfecture de la Gironde sur un dispositif de communication concernant de la sécurité routière. Distinguant, dans un premier temps, les notions de prise de risque passive et prise de risque active, l'auteur nous montre, dans l'attente des chiffres sur l'accidentologie qui ne seront connus que plus tard, comment les usagers de la route ont perçu cette campagne. Ici le risque routier est manifesté par d'emblématiques silhouettes noires, évoquant la mort, placées sur les axes les plus meurtriers. L'émotion est convoquée, organisée, le risque dramatisé comme dans de nombreuses autres campagnes de sécurité routière.

11 Évitant le registre de l'émotion et se réclamant de la Science, les experts jouent un rôle d'une importance capitale lorsque la perception de nouveaux risques se fait jour et qu'il s'agit de prendre des décisions dans un contexte d'incertitude. Rendre accessible les enjeux pour les citoyens, dégager la signification de la science, ouvrir le débat et ne pas opérer de simplifications réductrices, c'est l'attitude que préconise Olivier Laügt pour les médiateurs et les experts appelés en renfort des décideurs dans les périodes de crises. Si, par le passé de nombreuses décisions peuvent sembler avoir été confisquées et soustraites au débat public par les experts de toute nature au cours des crises que nos sociétés ont traversées, cet état de fait ne semble pas inéluctable. Selon cet auteur, une autre conception de l'expertise et de la communication en situation de risque pourrait 
prévaloir qui ne réduise pas la complexité mais s'efforce de la montrer, qui remette le débat démocratique et la négociation au cœur du phénomène.

12 C'est avec plaisir que nous vous proposons d'explorer plus avant ces pistes de réflexion, rapidement esquissées dans cette introduction. Que tous les auteurs qui ont contribué à ce numéro soient ici remerciés pour leur contribution à notre réflexion collective sur le thème de la communication du risque.

\section{NOTES}

1. Communication \& organisation, $\mathrm{n}^{\circ} 16,2^{\mathrm{e}}$ semestre 1999. Dossier coordonné par Michèle Gabay et Bruno Ollivier. 